\title{
A Wholistic Approach to Human-in-the-Loop Ecosystem
}

\author{
Anastasiia Prysyazhnyuk ${ }^{1}$ and Carolyn McGregor ${ }^{2,3}$ \\ Ontario Tech University, Oshawa, Ontario, L1G 0C5, Canada
}

\begin{abstract}
Space exploration is amongst the greatest endeavors of the humankind. It continues to fuel human curiosity and imagination, as the limits and boundaries continue to extend beyond the Low Earth Orbit to other destinations, such as Moon and Mars. Technological advancements and scientific discoveries continue to redefine the boundaries of the human body and mind, revealing remarkable resilience, cognitive, physical and psychological performance in austere environments. However, as human kind prepares to embark on deep-space missions, there are fundamentally new challenges and considerations that have to be addressed to ensure successful mission outcomes. Restricted space, increased communication delays and remoteness from Earth, with limited ability for emergency return, necessitate development of a comprehensive human-in-the-loop ecosystem, with increased autonomy and clinical decision-making capacity. The proposed research harnesses the potential of big data and streaming data analytics to support a paradigm shift from reactive to proactive health management in-flight. It demonstrates the potential to support prognostics, diagnostics and mitigation of medical contingencies in-flight through a meaningful and practical use of the acquired data to inform clinical decision making, significance of which is demonstrated within the context of adaption-based analytics in a ground-based study "Luna-2015".
\end{abstract}

\section{Introduction}

$\mathrm{O}$ ver the past century, revolutionary advancements have been made within the field of science and technology enabling the human race to explore other habitats within the solar system, marking the beginning of the era of human space exploration. Human curiosity has been fueled by the endless possibilities that lie beyond the low Earth orbit, which further motivated exploration of the solar system, but most importantly the exploration of the limits and possibilities of the human body and mind. Manned space exploration has provided an in-depth understanding of the human physiology and how the spaceflight environment impacts behavioral and health performance. The human capacity to effectively adapt to conditions of spaceflight, while maintaining occupational performance and critical decision-making has been well-documented in the scientific literature. ${ }^{1}$ However, as human kind prepares to embark on exploration-class missions to the Moon and Mars, there are fundamentally new challenges and considerations that have to be addressed to ensure successful mission outcomes. The psychological implications of human spaceflight are expected to amplify with increased distance from Earth, and very limited interpersonal and terrestrial communication, which may have a profound impact on astronaut health and performance. ${ }^{2}$ As such, it further necessitates the review of the human-in-the-loop ecosystem to ensure habitability, safety and comfort to support manned space exploration.

The existing human-in-the-loop ecosystem aboard the International Space Station (ISS) continues to evolve in response to technological improvements that have expanded human capacity to generate multi-type multi-source information. However, the latency in development of appropriate systems has failed to establish a harmonious humantechnology ecosystem, hindering the ability to effectively acquire, manage and leverage the enormous amount of data that is being produced. As a result, a vast amount of health, activity and environmental data has not fulfilled its potential, while very little fragments of that data have been practically used. Moreover, a significant amount of analytics, health and adaption-based analytics in particular, have been performed retrospectively, further contributing to impracticality of the acquired data. All while contributing to disintegration of an existing human-in-the-loop ecosystem, further separating the various components of the spaceflight environment that have been known to impact human health and performance. As such, the existing Crew Health Care System (CHeCS) on the ISS is a collection

\footnotetext{
${ }^{1}$ Graduate Student Researcher, Faculty of Health Sciences, Ontario Tech University and AIAA Student Member.

${ }^{2}$ Professor, Faculty of Business and IT, Ontario Tech University;

${ }^{3}$ Professor, University of Technology, Sydney, Australia and AIAA Member
} 
of the various components, rather than a wholistic, integrated system. The existing CHeCS has had limited ability to support prognostic and diagnostic capacity during space travel. ${ }^{3}$ Its health management strategy utilizes reactive care rather than a proactive health management approach. The existing health management approach presents a number of limitations to support health and safety of the crew on exploration-class missions, strongly emphasizing the need for a paradigm change.

In this research, we propose a novel framework that utilizes a big data approach, the enormous potential of which has not been leveraged by the field of space medicine yet, and as such, its potential value has remained in its infancy. The concept of big data is used to describe data in terms of three V's, it's volume, velocity and variety. ${ }^{4}$ The proposed framework extends the existing Artemis ${ }^{5}$ big data analytics platform, to provide an integrated framework architecture to support multi-variate data throughout all of the stages of its lifecycle, beginning with the data collection, all the way through to data reporting and storage within the context of space medicine. The proposed framework identifies the necessary modifications and extensions to a conceptual Advanced Crew Medical System (ACMS) Space Medicine Decision Support System (SMDSS) architecture, as outlined by the Canadian Space Agency, ${ }^{6}$ and the Artemis platform, in order to support real-time capability of health and wellness analytics, adaption-based analytics, activity and environmental analytics, all while ensure continuity of data acquisition, processing and storage. The proposed framework introduces a novel paradigm change that recognizes the dynamic nature of the data that can be effectively leveraged within the human-in-the-loop ecosystem. ${ }^{7,8}$ It further introduces a novel method of streaming data analytics, that has great potential to support the development of an autonomous medical decision support system, while contribute to action-taking and evidence-based clinical decision making within the field of space medicine.

The instantiations of the proposed framework will be demonstrated within the context of the ground-based Luna2015 study conducted at the NEK isolation habitation facility in Moscow, Russia. The near real-time functionality of the proposed analytics will be demonstrated within the context of adaption-based analytics, which utilize a re-modelled functional health state assessment algorithm that has been re-engineered as a stream-graph within the Online Analytics component of the proposed framework.

In summary, the research within this manuscript proposes a novel wholistic approach to the human-in-the-loop ecosystem, enabling integration of the various components of the Crew Health Care System to addresses existing challenges of retrospective discontinuous file-base data acquisition, in-batch data processing, extensive data downsampling and an enormous amount of data loss. It presents innovative solutions to support proactive prognostics, diagnostics and health management, while providing the necessary tools to support action-taking and informed decision-making within the field of space medicine.

\section{Related Work}

Medical monitoring in space is a set of highly structured and scheduled evaluations, performed at pre-defined time points throughout the mission to ensure safety, habitability, optimal health and performance of the crew. ${ }^{1}$ The practice of environmental and health monitoring in-flight greatly differs from pre- and post-mission evaluations due to significant physical constraints and specifics of the spaceflight environment, which are going to be further exacerbated by the hazards and challenges associated with deep space exploration.

Historically, the Crew Healthcare System (CHeCS) has supported medical monitoring aboard the ISS, encompassing three sub-systems, namely the environmental health system, health maintenance system and the countermeasures system. ${ }^{9}{ }^{10}$ While review of each of the CHeCS sub-systems is beyond the scope of this manuscript, the overarching limitations of existing in-flight monitoring approaches will be emphasized.

The existing health monitoring system aboard the ISS renders the human out-of-the-loop, by limiting accessibility and usability of the acquired data to inform health management and clinical decision-making in-flight. There has been a notable latency in the ability to access the acquired data either due to the architectural constraints of biomedical monitoring modalities, or due to lack of appropriate information systems to support real-time data acquisition, processing and analytics. Further accompanied by lack of interoperability between the CHeCS sub-systems, impairing the use of the acquired data in-flight. ${ }^{11}$ In summary, the acquired data has been subjected to retrospective file-based in-batch data processing, predominantly upon mission completion and return to Earth. As such, emphasizing an urgent need to develop a wholistic human-in-the-loop ecosystem to leverage the full potential of the acquired data to enable proactive health management in-flight.

It has been increasingly recognized that novel methods should be employed to enhance in-flight medical capability and inform the development of Space Medicine Decision Support System (SMDSS). SMDSS can be broadly defined as an information technology system that is able to effectively acquire and synthesize person-specific information, in such a way as to represent situation-specific information in a clinical context of interest. Moreover, it should be 
dynamic, provide real-time functionality and support practical, reliable and efficient use of technologies so as to enable in-flight medical data availability. ${ }^{11}$

Previously, the Canadian Space Agency highlighted the need in development of an Advanced Crew Medical System (ACMS), conceptual architecture presented in Figure $1 .^{8}$ The fundamental functionalities of such system have been summarized within three architectural modules, more specifically, Input, Data Processing and Handling, and the Output. The Input module identified the versatility of data types and sources, which are of relevance for health assessment within the context of spaceflight environment. All of which are meant to be integrated into a centralized electronic medical record (EMR) database, in preparation for consumption by the SMDSS, within the data processing and handling component. The Data Processing and Handling component comprises of the SMDSS, which in turn consists of a medical knowledge database and a decision-making engine. The Decision-Making engine has the capability to analyze incoming data and support prognostics, diagnostics and health state evaluations, the results of which are generated with the output module. While the proposed conceptual architecture of the ACMS SMDSS offers a novel wholistic approach to health assessments in space, it has some fundamental limitations. More specifically, it does not support real-time feedback functionality of derived analytics, further contributing to impracticality and lack of access to the medical data generated in-flight.

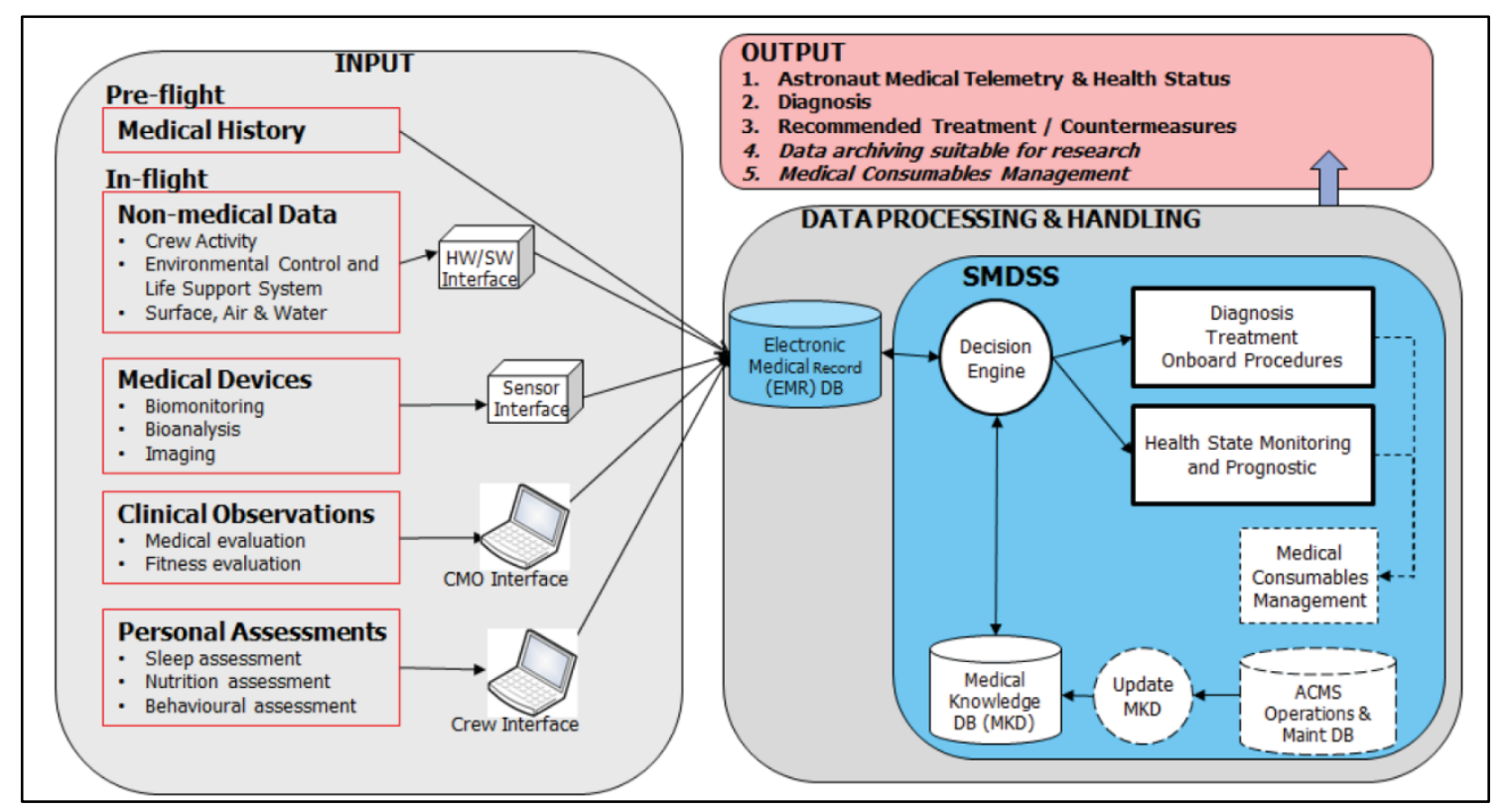

Figure 1. Conceptual diagram of the ACMS SMDSS architecture ${ }^{6}$

The concept of Big Data, which recognizes the volume, variety and velocity of the generated data has gained momentum across the various applications terrestrially, while it has remained in its infancy within the field of space medicine.

McGregor has previously proposed the Artemis big data analytics platform, for concurrent multi-stream, multipatient, multi-diagnosis temporal real-time analysis. ${ }^{5}$ Artemis is provisioned as a cloud-based health analytics as-aservice platform that supports autonomous, remote health monitoring through integration of the relevant physiological, environmental and activity data acquired with a range of sensors and devices. Figure 2 presents the architectural components of the Artemis platform, which include Data Collection, Data Acquisition, Online Analytics, Data Persistency, Knowledge Extraction, (re)Deployment and Results Presentation, described in detail in 5, 12, 13. Overall, the Artemis platform is a state-of-the-art platform that has the potential to support complex health analytics in remote and austere environments. Its framework has been specifically designed to support autonomous, remote functionality that would be well-suited for clinical decision support systems aboard the spacecraft, especially for exploratory-class missions where there will be significant communication delays and extremely limited ground-based medical support. However, Artemis has been initially designed for physiological data streams and has incompatibility issues with existing biomedical monitoring modalities used in-flight. 


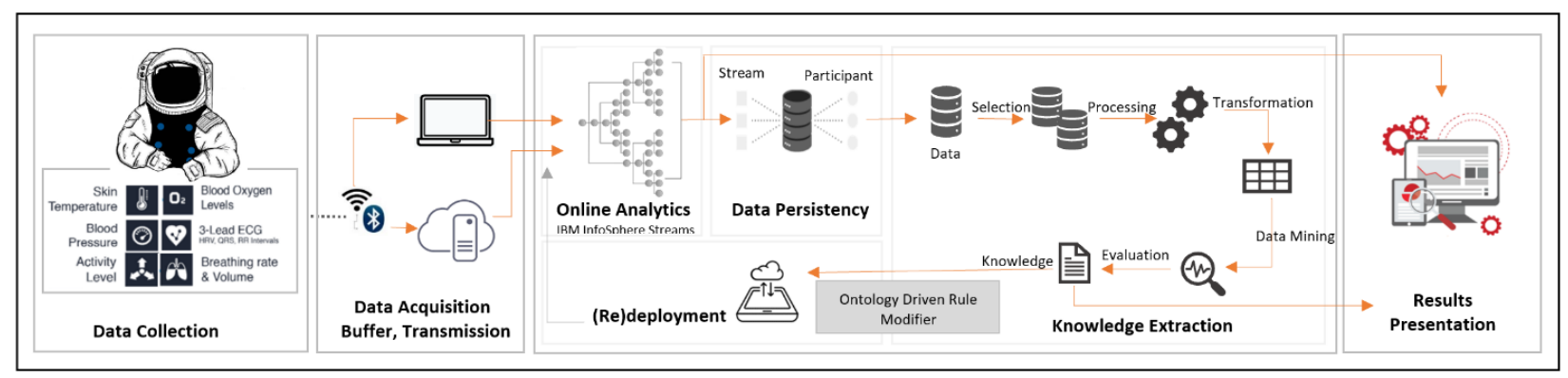

Figure 2. Architectural components of Artemis health analytics as-a-service for spaceflight [12].

This research recognizes that the conceptual ACMS SDMSS architecture and prior Artemis platform can be merged, with the introduction of a few extensions to address existing limitations and support the development of wholistic human-in-the-loop ecosystem for real-time in-flight health monitoring. It further supports interoperability of the different sub-systems, compatibility of biomedical monitoring modalities and their respective information technology systems, while enables integration and linkage of relevant data types for real-time analytics.

\section{Proposed Framework}

The proposed big data analytics framework is made up of eight core components, which are the data collection, data capture, data integration, data persistency, online analytics, knowledge discovery, (re)deployment and results presentation, schematically represented in Figure 3 and described in detail in ${ }^{7}$.

The components that have been adopted from prior Artemis research include Data Persistency, Knowledge Discovery, (re)Deployment, Online Analytics and the real-time streaming adaptive API of the Data Integration component. The extensions presented in the proposed framework include Data Collection, Middleware Data Capture, file-based data integration and Results Presentation components. ${ }^{7,8}$

The data collection component supports acquisition of multi-source multi-variate data, including non-medical data, more specifically environmental and activity data, supplemented with medical history, physiological data, clinical observations and personal assessments.

The data capture component presents a queueing mechanism that generates various queues according to the data format, such as the data streams and the file-based data packets, which are consumed by the data integration component. Within the data integration component, the incoming data streams and file-based packed of data are subjected to a collection of data processing steps, including parsing, filtering and enrichment, which result in linkage of the relevant data, in preparation for subsequent analytics.

The Online Analytics component utilizes the stream computing paradigm to support near real-time and/or realtime functionality. As such, the stream graphs of interest are adaption-based analytics, environmental and activity analytics, health and wellness-based analytics.

The proposed framework also has the capacity to support retrospective knowledge discovery through utilization of temporal data mining and artificial intelligence based deep learning methods. Moreover, the (re)deployment component supports updating of existing stream graphs, within the same mission, and/or deployment of new stream graphs, which have demonstrated clinical validity. It should be noted that data buffering mechanisms are integrated within various components of the proposed framework to ensure fault-tolerance and persistence of data warehousing of raw data and derived analytics.

Lastly, the results presentation component supports the development of interactive dashboards with multiple interface functionality that can be customized in accordance with specific user access controls.

The proposed framework recognizes that novel space medicine systems must be dynamic, provide real-time functionality and be able to integrate inputs from variety of data sources, and as such, contribute to a valid, reliable and practical use of technologies to support successful manned space exploration.

The clinical significance of the proposed framework will be demonstrated within the context of adaption-based analytics, as part of the terrestrial demonstration, namely the "Luna 2015" study, which will be detailed in the section to follow. 


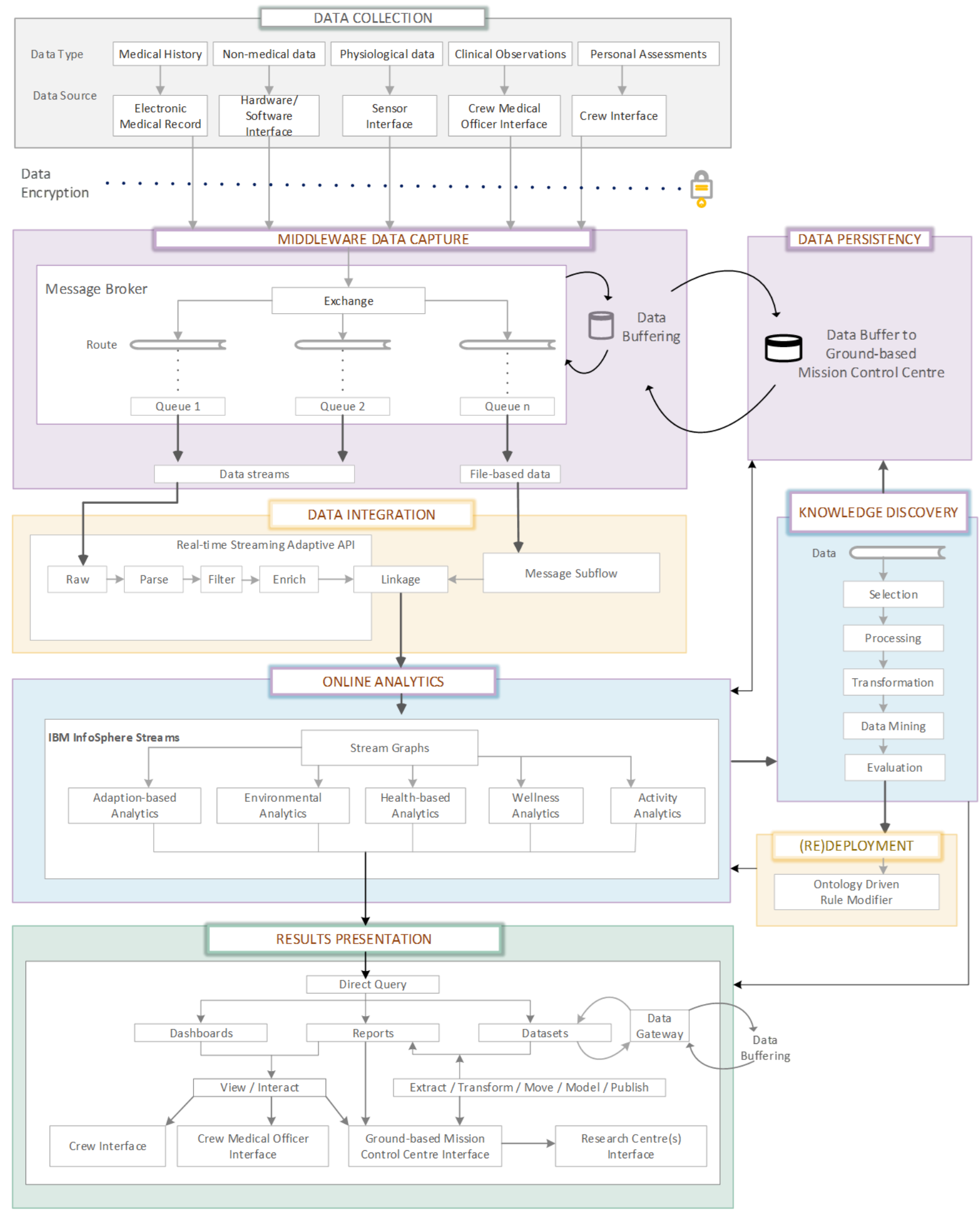

Figure 3. The proposed framework for a wholistic approach to human-in-the-loop ecosystem ${ }^{7}$ 


\section{Terrestrial Demonstration}

The proposed framework has been instantiated within the context of the ground-based study "Luna 2015", in which the clinical significance of a wholistic human-in-the-loop ecosystem has been demonstrated on the basis of adaptionbased analytics.

"Luna 2015" was the first of its kind all-female isolation and confinement study conducted at the Nazemnyy eksperementalnyy komplks (NEK) / Ground-based experimentation facility located in Moscow, Russia. The study cohort consisted of six female participants, between the ages of $30 \pm 5$ years old, with an average height of $163 \mathrm{~cm}$ and mean weight of $58.5 \mathrm{~kg}$. The overarching objective of the research was to investigate physical and psychological performance of the all-female crew on a seven-day simulated flight around the Moon. This research study was approved by the IBMP Research Ethics Board, and the Ontario Tech University Research Ethics Board under REB\# 15-047 Integration of Russian Cosmonaut Monitoring with Artemis and Artemis Cloud.

Data collection was performed in accordance with the ISS protocol, and was scheduled and discontinuous. The Holter-style ECG monitoring device, Cosmocard, currently in use within the Russian segment of the ISS, was utilized for acquisition of electrocardiogram recordings. ECG-recordings spanned over the period of 24-hours and were collected for half of the crew on the second, fourth and sixth day of the study, and similarly on the third, fifth and seventh day of the study for the remainder of the crew. ECG sampling rate was set to 500 samples/second. Following each instance of data collection, the device was docked to support data acquisition and transmission. The outlined data collection approach presented fundamental limitations to the approbation of the proposed framework, as such the data had to be re-played, to simulate real-time data acquisition.

The adaption-based analytics were performed by a traditional retrospective approach and through instantiation of the functional health assessment as an instance within the Online Analytics component. The functional health assessment utilizes step-wise discriminative analysis to extract the various geometric, statistical and spectral heart rate variability (HRV) indices from electrocardiogram recordings. The HRV indices of interest, include heart rate, stress index, number of normal-normal (NN) intervals differing by more than $50 \mathrm{~ms}$ and the high frequency band of the total spectral power. The HRV indices are then used to compute two canonical variables, $\mathrm{L}_{1}$ and $\mathrm{L}_{2}$, used to establish a phase plane of functional states. The efficacy of both instantiations, the traditional approach and instantiation within the proposed framework was compared and schematically summarized in Figure 4.

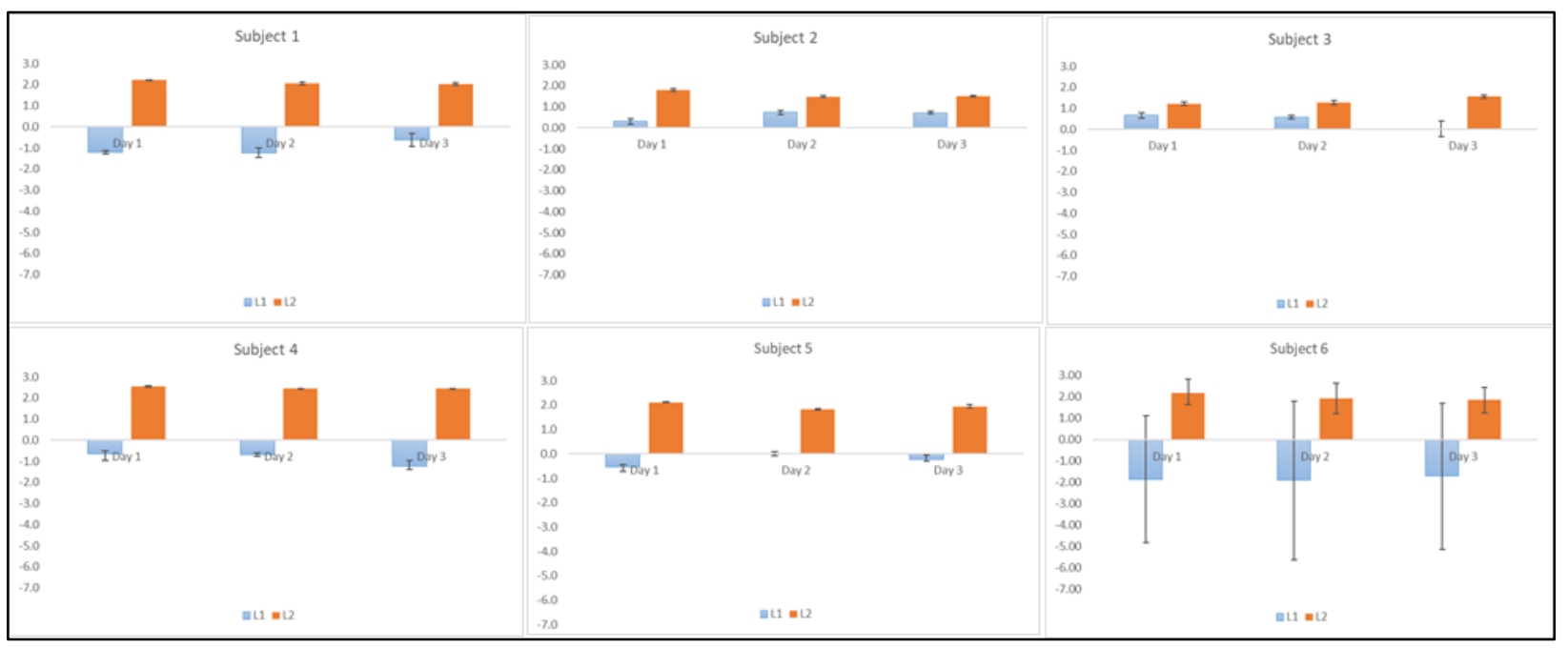

Figure 4. Efficacy of algorithm instantiation with the proposed framework.

The efficacy of algorithm instantiations was assessed by computing the average daily values and their respective standard deviations. The computed $\mathrm{L}_{1}$ and $\mathrm{L}_{2}$ values for Subjects 1 through 5 were statistically significant and accurate, supported by a low standard deviation. The minor variance observed between the values was attributed to the specifics of QRS complex and R-peak detection algorithms of the respective instance of the functional health state assessment. The reported results for Subject 6 introduced a great level of variance due the poor quality of the raw ECG signal, supported by high values of standard deviation. Further to that, geometric feature variance was observed and mainly attributed to the specifics of the ECG processing approach and R-peak detection, which was significantly impacted by the poor quality of the raw signal. As such, the geometric feature variance and its respective assigned "weight" 
within $L_{1}$ calculation explained the large discrepancy in $L_{1}$ values for Subject 6. Overall, the proposed instantiation of the functional health state algorithm within the Online Analytics component has demonstrated great potential to support adaption-based analytics, while provide statistically significant accuracy and validity of the produced results. Further iterations of the proposed algorithm would necessitate improvement of the QRS complex detection algorithm, especially for processing of contaminated data samples.

The overall adaption-based assessments are presented in a traditional format, as a phase plane of functional states, displayed in Figure 5, where four main functional states can be distinguished. More specifically, the state of physiological norm, prenosological, premorbid and the state of pathology. The transition between the state of physiological norm to pathology occurs as a result of stressor exposure, which contributes to elevated systems tension and depletion of functional reserves, in an attempt to preserve homeostasis.
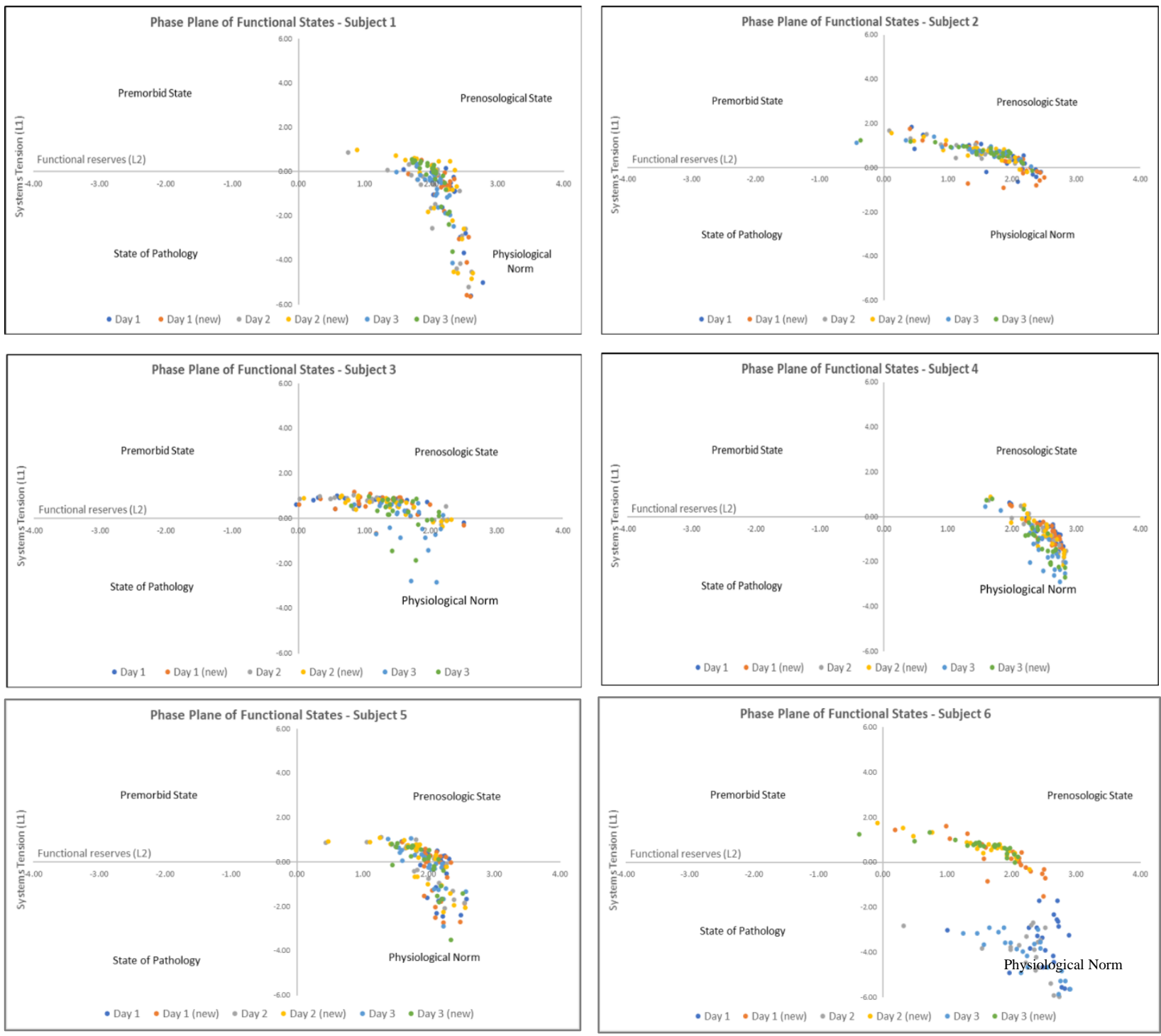

Figure 5. Adaption-based analytics, as a phase plane of functional states, for Subject 1-6 during Luna 2015.

The functional health states are presented as aggregated hourly values for each 24 -hour dataset computed by two instances of the functional health state algorithm, on an individual basis. The traditional instantiation is denoted by the day number (i.e. Day 1, 2, 3), while the instantiation within the Online Analytics component of the framework is denoted by the day number supplemented with "new" (i.e. Day 1 (new), Day 2 (new), Day 3(new)). As denoted within Figure 5, the activity of adaption mechanisms was dynamic throughout the entire duration of the study, while characteristics behaviours could be observed due to time-specific or mission-specific tasks that have been performed. 
While all study participants remained within the boundaries of physiological norm or just on the verge of Prenosological states, there has been a significant variation in the levels of experienced systems tension. As such, this approach demonstrated great potential to support early detection monitoring and understanding of physiological and psychological responses that occur as a result of specific tasks or activities. This information can be harnessed to support the development of personalized resilience strategies and mitigation of spaceflight-related stressors, so as to preserve cognitive, psychological and physiological well-being of the crew and successful execution of mission objectives.

\section{Conclusion}

Technological and scientific advancements continue to enable safe prolonged human presence in space, enabling exploration of deep space habitats, such as the Moon and Mars. Deep space exploration is expected to present fundamentally new challenges, which are vastly different from the majority of manned spaceflight experience known to-date. The potential risks, challenges and spaceflight hazards will amplify, placing a significant emphasis on the development of new, innovative solutions that could support autonomy and inform clinical decision-making in-flight. It emphasizes an urgent need to support the paradigm change from reactive to proactive prognostics, diagnostics and health management during deep space exploration, while ensure that human is an integral part of the humantechnology ecosystem aboard the spacecraft.

This manuscript exposed significant latency in the development of appropriate information technology systems, which limit accessibility and usability of the acquired data in-flight, while the cumbersome and disintegrated data processing protocols continue to contribute to extensive data smoothing and an enormous amount of data loss. Further emphasizing the existing challenges of retrospective file-based in-batch data processing methods and techniques, utilized within the Crew Healthcare System aboard the ISS.

The proposed research harnesses the enormous potential of big data and streaming analytics in an attempt to develop a harmonious, wholistic human-in-the-loop ecosystem, which can leverage the power of artificial intelligence and machine learning to support dynamic, real-time functionality and enable practical, reliable and efficient use of technologies in-flight.

The clinical significance of the proposed big data framework is demonstrated on the basis of adaption analytics within the context of terrestrial "Luna 2015" study. The Luna 2015 case study addressed the existing challenges of retrospective analysis of file-based packets of data and the tremendous data down-sampling that occurs during traditional processing of functional health state assessment. The instantiation of the proposed framework further demonstrated great potential to enhance early detection monitoring and inform clinical decision making in-flight. Thereby, contributing to practical and meaningful use of physiological data to support proactive prognostics, diagnostics and health management during space flight. The end-to-end attestation of the proposed framework will be the subject of future studies, so as to demonstrate its potential as an autonomous clinical decision support system in space medicine. It will further demonstrate its capacity to support real-time health analytics, clinical discovery and improve early detection of maladaptive responses during spaceflight.

\section{Acknowledgments}

This research has been supported by the Canada Research Chairs program (\#950-203427 and \#950-225945) and the Canadian Foundation for Innovation (\#203427).

\section{References}

${ }^{1}$ Nigossian A., Mohler S., Gazenko O., and Grigoriev A. (2004). Space biology and medicine - volume IV, Health, Performance and Safety of Space Crews. American Institute of aeronautics and Astronautics. Pp.1-85.

${ }^{2}$ Bizzari, M., Masiello, M.G., Guzzi, R., and Cucina, A., 2017. Journey to Mars: A Biomedical Challenge. Perspective on future human space flight. Organisms: Journal of Biological Sciences, 1(2): pp.15-26.

${ }^{3}$ Cermack, M., 2006. Monitoring and telemedicine support in remote environments and in human space flight. British Journal of Anaesthesia, 97(1), pp.107-114.

${ }^{4}$ Wang, Y., Kung, L., \& Byrd, T. (2018). Big data analytics: Understanding its capabilities and potential benefits 
for healthcare organizations. Technological Forecasting and Social Change, 126, 3-13.

${ }^{5}$ McGregor C., (2013). A platform for real-time online health analytics during spaceflight. IEEE Aerospace Conference Proceedings. doi:10.1109/aero.2013.6497382.

${ }^{6}$ RFI regarding the Advanced Crew Medical System (ACMS) Space Medicine Decision Support System (SMDSS). (2016). Retrieved 16 October 2020, from https://buyandsell.gc.ca/cds/public/2016/07/21/ce21b3d9c1ab2b55122a526b38eea330/ABES.PROD.PW_MTB.B54 5.E13964.EBSU000.PDF

${ }^{7}$ Prysyazhnyuk, A., and McGregor C., 2020. A wholistic approach to assessment of adaptation and resilience during spaceflight, $71^{\text {st }}$ International Astronautical Congress, CyberSpace Edition.

${ }^{8}$ Prysyazhnyuk, A., and McGregor, C., 2020. Adaption-based analytics for assessment of human deconditioning during deep space exploration, 2021 IEEE Aerospace Conference, Big Sky, Montana, USA.

${ }^{9}$ Holland, D. and Kemmerling, C., 2000. Some Medical Issues and Technologies for Preserving Human Performance during Long-Duration Spaceflight. Proceedings of the Human Factors and Ergonomics Society Annual Meeting, 44(28), pp.546-549.

${ }^{10}$ Hamilton, D., Smart, K., Melton, S., Polk, J. and Johnson-Throop, K., 2008. Autonomous Medical Care for Exploration Class Space Missions. The Journal of Trauma: Injury, Infection, and Critical Care, 64(Supplement), pp. S354-S363.

${ }^{11}$ Steinkraus, L., B. Rayman, R., Butler, W., Marsh, R., Ercoline, W. and Cowl, C., 2012. Aeromedical Decision Making - It May Be Time for a Paradigm Change. Aviation, Space, and Environmental Medicine, 83(10), pp.1006-1007.

${ }^{12}$ Prysyazhnyuk, A., McGregor, C., Chernikova, A., \& Rusanov, V. (2019). A sliding window real-time processing approach for analysis of heart rate variability during spaceflight. In 70th International Astronautical Congress. Washington, DC, USA.

${ }^{13}$ McGregor, C., Orlov, O., Baevsky, R., Chernikova, A., V. Rusanov, "Big Data Analytics for Continuous Assessment of Astronaut Health Risk and Its Application to Human-in-the-Loop (HITL) Related Aerospace Missions", AIAA SciTech Conference, Grapevine, Texas, Jan 2017, 7 pages. 